\title{
移动大数据时代: 无线网络的挑战与机遇
}

张平，崔琪楣"，侯延昭，徐瑨

北京邮电大学, 北京 100876

* 联系人, E-mail: cuiqimei@bupt.edu.cn

2014-11-03 收稿, 2014-12-19 接受, 2015-01-20 网络版发表

国家自然科学基金(61471058, 61421061)和北京高等学校青年英才计划(YETP0429)资助

摘要 随着移动互联网、云计算、物联网、机器类型通信等新兴信息通信技术的飞速发展，信 息社会进入了网络化的大数据时代. 快速普及的智能化移动终端应用助推了全球移动数据流量 的大幅度增长. 在移动大数据时代, 海量数据、业务类型演进、数据多样化、数据空-时域分布 不均匀等特征给无线网络带来了严峻的挑战. 为了应对挑战, 一方面, 无线网络从新频谱拓展、 传输技术、智能立体化组网等多维度进行演进以满足大数据传输与应用的需求; 另一方面, 移 动大数据作为一种新的生产要素改变着人们认知网络的方法, 无线网络可以充分借鉴互联网数

关键词 移动大数据 无线网络 挑战与机遇 网络部署 资源管控预测 据挖掘的理论与方法, 实现网络的灵活部署、无线资源的优化配置和低能耗绿色通信.

当今, 移动互联网、云计算、物联网、机器类型 通信等新兴信息通信技术的飞速发展导致数据流量 的爆炸式增长和数据结构类型的高度复杂化, 信息 社会进人了网络化的大数据(big data)时代 ${ }^{[1,2]}$. 宏观 上大数据即指海量的数据; 从微观上定义, 大数据是 指需要通过快速获取、处理、分析以从中提取有价值 的海量、多样化的交易数据、交互数据与传感数据等 数据, 其主要特征包括: 海量 (volume)、类型繁多 (variaty)、增长迅速(velocity)、价值巨大(value)等 ${ }^{[3]}$. 随着智能手机、平板电脑等智能化终端的快速普及, 移动数据流量和信令呈现非线性指数增长趋势, 将 逐渐成为大数据的主体, 这对无线网络的演进与发 展产生重要的影响.

在移动大数据时代下, 无线网络的挑战与机遇 并存. 一方面, 未来无线网络需具备承载大数据的能 力; 另一方面, 有效地利用海量数据中的离散信息可 充分实现网络的潜在价值. 基于上述考虑, 本文介绍 了移动大数据的主要特征, 分析了其给无线网络带 来的主要挑战和发展机遇.

\section{1 移动大数据的主要特征}

\section{1 海量数据}

在信息社会，人、机、物之间的高度融合与互联 互通激发了海量数据的涌现. 思科统计显示 ${ }^{[4]}$, 到 2013 年底, 全球移动数据流量为 $1.5 \mathrm{~EB} /$ 月, 接近 2000年全球互联网总流量的 18 倍，预计到2018年总 流量将增长至 $15.9 \mathrm{~EB} /$ 月(图1). 同时，快速普及的智 能化移动终端应用助推了全球移动数据流量的大幅 度增长. 以智能手机为例, 截止到2013年底, 智能手 机的数量仅占全球在用手机总量的 $27 \%$, 但其产生的 流量占全球手机总流量的 $95 \%$ (每部智能手机的移动 数据流量为 $529 \mathrm{MB} /$ 月).

\section{2 业务类型演进}

随着移动互联网应用的发展，传统蜂窝网络所 承载的业务正在由传统语音、短信向多样化的具有互 联网特征的新业务类型拓展.例如，微信等即时通讯 类业务、社交网站和搜索引擎等交互类业务、在线视

引用格式: 张平, 崔琪椐, 侯延昭, 等. 移动大数据时代:无线网络的挑战与机遇. 科学通报, 2015, 60: 433-438 


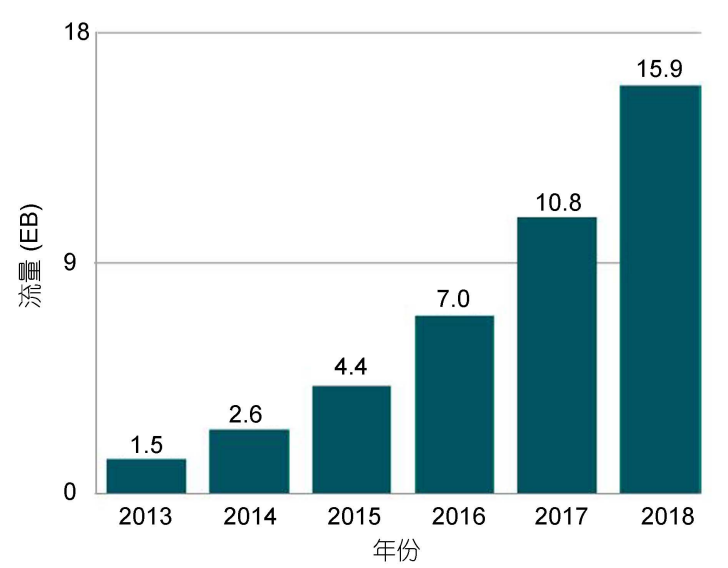

图 1 思科预测全球数据流量 ${ }^{[4]}$

Figure 1 Global mobile data traffic forecast-Cisco ${ }^{[4]}$

频和在线音乐等流媒体业务等. 新业务继承互联网 的特征，而传统无线通信网在通信机制、互联互通规 则等方面与互联网有完全不同的设计理念, 难以适 应新业务的需求. 例如, 以即时通信类业务为代表的 小包持续性突发实时在线业务类型，其包含频繁的 文本、图像信息和周期性的pings, 这导致无线网络在 连接和空闲状态间进行频繁的切换, 不仅增加设备 的能耗, 还造成严重的信令开销, 使得资源利用率十 分低下. 然而, 在移动互联网业务中, 即时通信业务 的比例日益增高. 如图 2 所示 ${ }^{[3]}$, 截至2014年6月底, 我国手机即时通信网民数为 4.59 亿, 比 2013 年底增长 了 2842 万，半年增长率达 $6.6 \%$; 手机即时通信使用 率为 $87.1 \%$, 比 2013 年底提升了 1 个百分点.

\section{3 数据多样化}

海量的在线数据, 将引入新的计算、存储方式, 网络业务将呈现不同的特征和属性, 而移动数据类

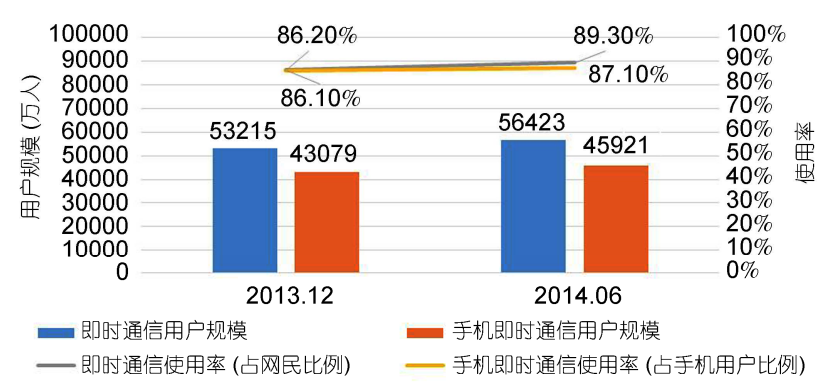

图 2 2013-12 2014-06 即时通信/手机即时通信用户规模及使用率 ${ }^{[3]}$ Figure $2 \mathrm{IM} /$ mobile phone IM subscribers and usage ${ }^{[3]}$
型更加繁多, 包括结构化数据、半结构化数据和非结 构化数据. 现代移动互联网产生了大量非结构化数 据, 包括各类视/音频信息、办公文档等, 其在数据类 型中所占比例呈现升高态势. 根据Gartner Group统 计, 如今 $80 \%$ 的数据为非结构化数据 ${ }^{[5]}$, 而移动互联 网中这一比例已达到 $95 \%$ 以上. 大量非结构化的数据 随机散落于不同的智能终端中, 其数据格式互不兼 容, 读取和存储具有随机性, 这对于系统的传输带 宽、控制信令开销、资源分配等带来了严峻挑战.

另一方面, 在无线接人网络侧可获得多种特征 的大数据. 在物理层可获得信号强度、信噪比(SNR)、 用户接人位置(中心/边缘)、多普勒(Doppler)速度等具 有典型无线特征的数据信息，在媒体接人控制层 (MAC) 可获得用户级别、请求速率、调度优先级、单 次接人时延(如QQ和下载应用)等具有服务质量特征 的数据信息, 在应用层可获得用户业务习惯(如平均 通话时长)、用户感知体验(如网络容忍度)、用户套餐 (如付费习惯、续约习惯、消费分析) 等具有用户行为 特征的数据信息. 如何有效地利用海量多样化的大 数据, 挖掘其价值服务于网络是未来值得研究的重 要内容.

\section{4 数据的空-时域大动态变化}

用户的随机趋同性使得网络的业务密度分布在 空-时域上呈现不均匀的特性，热点区域业务量占 $70 \%{ }^{[2]}$. 图3是来自于某移动运营商现网实测话务数 据样本的业务分布图, 数据流量的密度呈现空-时域 非均匀特性. 空间域上，城市中心的局部地区业务量 超大，而城市边缘地区数据业务量却低于平均水平; 时间域上, 数据流量的变化剧烈, 工作时间商务区数 据流量大而居民区数据流量小，休息时间则刚好相 反. 数据流量在空-时域上的大动态变化使得无线网

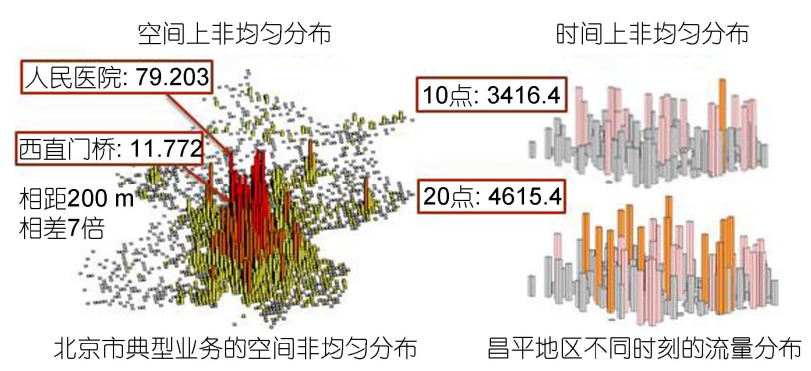

图 3 数据业务的空-时特性

Figure 3 Data traffic characteristics in time-space domain 
络在站点部署、热点覆盖、资源分配等问题上的灵活 性和智能性需求更加迫切.

\section{2 移动大数据对无线网络的挑战}

如前所述, 移动大数据的涌现对无线网络提出 了更高的需求：海量数据要求网络具有更大的承载 能力; 业务类型演进和数据多样化要求资源管理策 略更加智能高效; 数据空-时域分布不均匀要求网络 部署更加合理等. 为应对移动大数据的冲击, 无线网 络节点不再扮演单纯转发的角色，而要同时具备计 算、存储、分析、决策、动态和智能化的功能. 因此, 移动大数据时代的到来, 在网络部署、资源管控、移 动通信安全、网络能耗等多方面对传统无线网络提出 了诸多挑战.

\section{1 网络部署}

移动数据流量和信令均呈非线性高速增长, 给 无线接人网络带来巨大压力. 超密集化的小小区 (small cell) 部署被认为是应对海量数据增长和数据分 布不均匀的最有效手段 ${ }^{[6]}$ ，小小区增强已成为 $3 \mathrm{GPP}$ LTE-Advanced Release 12中最重要的候选技术 ${ }^{[7 \sim 9]}$. 与此同时, 受限于低频范围内宽带连续频谱资源极 度贾乏，未来 $5 \mathrm{G}$ 网络将不得不向更高频段扩展。由 于小小区部署主要针对室内热点区域, 高频段无线 信号传播的特征(高损耗、低穿透)恰好可以削弱小小 区与其他小区之间的干扰. 因此, 室内高频段小小区 部署将是未来超密集化网络部署的关键问题.

严重的信号衰落及信号传播通常在视距范围内, 使得室内小区形状呈现“准”确定性和小区边缘锐利 化的特征, 这对室内网络覆盖规划引人了难题. 室内 节点的密集程度主要受建筑物布局的影响, 若以最 小化室内无线节点为网络规划目标，锐利的小小区 边缘会使问题建模异常复杂.

\section{2 资源管控}

新型业务类型的不断涌现, 数据多样化和流量 空-时域大动态变化给传统资源管理体系带来了新的 难题. 目前的资源管控策略是既定且自发的，资源分 配方式依赖于网络的当前负载和拥塞状态、终端业务 请求等, 使得全局的资源管控效率较低：从资源管控 的目标看，以网络吞吐量为主体的优化目标无法实 现基于大数据业务归类的差异化控制目标，导致管
控策略灵活性低，用户体验差；从资源管控的约束条 件分析, 数据多样化和需求异质化导致资源管控的 维度增加且各维度间不对称，资源管控优化策略的 制定和优化目标的求解变得异常复杂. 其次, 对于继 承了互联网设计理念的新业务类型 $(\mathrm{QQ}$ 、微信等), 在 当前的移动网络中针对各种不同业务(如不同的传输 速率、业务达到间隔、时延等)只设计了一种面向连 接的信令/控制机制，对于突发性业务会导致相对较 高的信令开销; 同时, 相对于移动互联网新业务的不 断创新, 资源管控的智能化进程滞后, 使得用户附着 度较低. 最后, 传统移动网络资源管控主要是基于以 小区为中心的均匀设计理念, 且总是针对网络最差 的状态进行宏观的资源配置, 这与实际系统中海量 业务空-时域的不均匀分布特征不符，网络资源管理 须从以小区为中心向以用户体验为中心、以数据为中 心转变.

\section{3 数据安全性}

数据安全 ${ }^{[10,11]}$ 一直是无线网络中备受关注的核 心问题. 在移动大数据时代, 用户接人更加频繁、灵 活．当用户在 $Q Q$, Facebook等社交网络上分享信息 时，大量个人信息不仅存储在终端里，也分散在互联 网上. 恶意用户利用大数据分析技术处理这些海量 数据, 更容易窃取他人隐私, 甚至可以针对用户进行 全景观察，通过对用户所有数据的分析了解各因素 之间的关系, 判断用户的状态、预测用户行为 ${ }^{[12]}$. 除 了用户隐私安全之外，数据的访问控制和可信度也 面临挑战. 用户属性和访问需求的多样化使保密权 限设置更加困难. 数据源伪造(伪基站、钓鱼 $\mathrm{WiFi}$ 等)、 数据篡改、数据窃听和数据失真造成数据可信度 降低.

与有线通信相比，无线通信固有的广播特性和 传输媒介的开放性使其更易受到窃听等安全攻击. 而且，未来无线网络呈现网络架构扁平化、信息交 互IP化、异构融合等趋势，一方面为无线通信带来 更多来自无线网络以及互联网的安全威胁, 另一方 面, 安全攻击更容易扩散并产生较大影响。随着大 数据分析能力的增强, 单纯依赖高层安全措施已不 足以保障无线通信安全, 特别是网络节点数目增 多、随机性增强使高层安全措施的部署愈发复杂和 困难. 因此，应充分结合无线通信的基本特征，利 用信道编码、信号处理等技术从物理层人手搭建无 
线通信的第一道安全屏障, 即物理层安全 ${ }^{[13]}$. 该技术

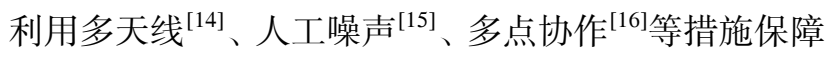
无线传输安全, 但是, 将消耗部分网络资源, 牺牲系 统容量、能效性能. 因此, 安全性与其他性能指标的 合理折中也是亟待考虑的问题.

\section{4 高能耗}

在全球能源匮乏和建设资源节约型社会的背景 下, 高能耗问题已经成为制约信息通信行业发展的 瓶颈问题之一. 移动网络的能耗主要分为两部分: 网 络侧能耗(图4)和移动终端能耗. 其中, 前者所占比 重较大, 其能耗在不同层面的分布比例如图 4 所 示 $^{[17]}$, 基站是最主要的耗能部分, 其能耗占总能耗 的一半以上. 据统计, 中国通信网基站的年消耗电量 超过200亿度，2012年中国联通缴纳的电费超过了其 员工薪酬及福利开支的 $1 / 3^{1)}$.

在大数据背景下, 用户在享受 $Q Q$ 、微信、社交 网站、搜索引擎、在线视频等新业务的同时, 无论在 网络侧还是终端侧均需要消耗更多的计算、存储、能 量等资源. 在无线接人网络侧, 超密集化的网络站点 部署会显著加剧高能耗问题 ${ }^{[18]}$. 在终端侧, 由于终 端能耗在总耗能中的比例较低, 在过去的几十年, 终 端能耗不被重视, 终端节能技术主要考虑如何延长 其待机时间. 然而, 随着智能终端的迅速增长, 今天 已步人后摩尔时代 ${ }^{[19]}$, 多样化的业务应用增加了智 能终端负载与日平均使用率, 这导致移动终端能耗 成为迫切解决的关键难题.

\section{3 移动大数据对无线网络带来的机遇}

为了应对移动大数据给无线网络带来的前述挑 战, 无线网络将从资源、传输、组网 3 个方面不断演 进, 以满足用户对传输速率和业务质量的更高要求. 例如, 挖掘毫米波频段、通过认知的方式提高频谱资 源的利用率; 引人新的传输技术, 通过大规模天 线、同时同频全双工及新型编码调制方案等提高系统 的传输速率; 组建智能立体化网络, 实现不同应用场 景下的人与人、人与物、物与物的立体化接人.

与此同时, 快速地从价值密度低的海量数据中 分析提炼出有效信息能进一步优化无线网络. 初期 的无线网络演进, 以提升网络整体的容量为目标, 是
一种以网络为中心的演进思路; 随着移动通信的发 展, 用户的主观体验成为移动网络优化的主要目标, 网络将从以网络为中心向以用户为中心演进; 而随 着大数据时代的到来, 海量数据已经成为移动网络 的核心财富, 使得无线网络向着以数据为中心的方 向发展.

\section{1 基于大数据挖掘的网络部署}

如前所述, 海量的移动数据具备较高的空-时变 化特征, 传统固定的移动网络部署无法有效匹配某 一特定区域内的流量与负载特征，超密集化的小小 区部署也无法实时适配业务需求的动态变化, 从广 义角度而言, 移动大数据的出现需要无线网络部署 由静态转变为动态、由固定配置转变为灵活配置. 为 解决此问题, 中国移动提出了 C-RAN(centralized, co-operative, cloud-RAN)架构 ${ }^{[20]}$, 将多个基带汇聚形 成大覆盖下的 “大基站”, 多个覆盖区域内的无线射 频单元共享基带设备和资源, 可实现计算资源的按 需有效分配. 这在一定程度上体现了以数据为中心 进行网络部署的思想. 进一步地, 以 SDN(software defined network)和NFV(network function virtualization)为代表的无线虚拟化技术能够实现基于业务特 征的网络协议动态部署 ${ }^{[21]}$, 通过对网络节点抽象和 统一调配, 网络的部署和管控更加灵活.

与传统的网络部署方式不同, 在以数据为中心的 网络动态部署中, 移动大数据充当决策者或主导者的 作用. 数据的多维特征决定了节点和协议的部署方式, 这有助于无线网络拓扑结构的优化，业务特征的动态 匹配, 并能提高无线网络的整体资源利用水平.

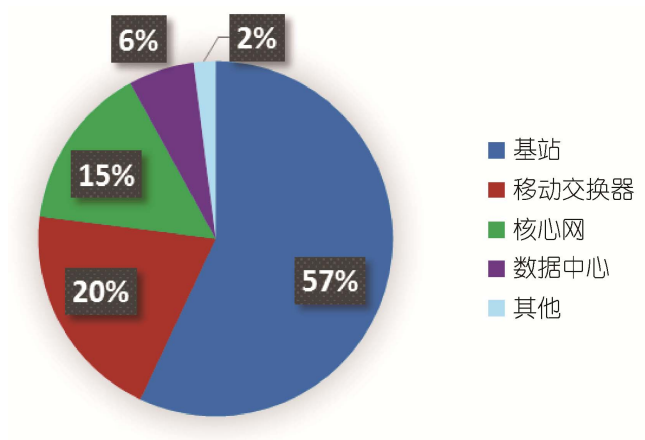

图 4 网络侧能耗分布图 ${ }^{[17]}$

Figure 4 Energy consumption distribution in network side ${ }^{[17]}$

1) 中国联合网络通信股份有限公司 2013 年半年度报告 


\section{2 基于大数据挖掘的无线网络资源精准投放}

海量数据在空-时域分布不均匀的特性对无线网 络中的资源调度与分配提出了更高的要求, 对大数 据中蕴含的抽象信息进行分析和归纳, 挖掘规律性 并预测业务走势, 有针对性地进行资源分配, 能为未 来无线网络提供新的发展契机.

首先, 基于数据挖掘的聚类分析 ${ }^{[22]}$ 能有效完成 相同特性的小区聚类，便于针对不同特性的小区进 行特定的资源分配, 如基于经纬度、网络覆盖和业务 分布特征进行小区聚类，从而指导资源精准投放和 业务推送.

其次, 随着移动数据业务用户数量的增多和需 求的差异化, 用户出现等级分化, 无线网络中的策略 控制与计费(PCC)方式需进一步考虑用户的匹配性， 从而制定更合理的资源分配方式以适配不同层级的 用户体验. 大数据可以应用于PCC, 通过分析用户特 性、业务特性以及对用户的行为习惯进行预测，从而 对资源进行合理的分配与控制，提高PCC的效率，提 供差异化服务，提升无线网络智能管控水平.

再次, 海量数据挖掘能够实现以用户体验 $(\mathrm{QoE})$ 为导向的资源精准投放. 未来无线网络对QoE的研究 需兼顾环境因素、用户因素及服务因素, 考虑混合多 业务场景下的个性化资源分配和管理. 对QoE的评价 方法也不仅仅限于客观评价或主观评价，伪主观评 价因既有主观评价与用户感知一致的特点, 又有客 观评价方法简便、可实时应用、可移植的优势，从而 成为更贴合用户需求的评价方法 ${ }^{[23]}$ ，但其所需要的 数据量远高于前两种. 基于大数据的海量数据分析 能为有效评价用户体验提供充分的信息, 从而有力 支撑伪主观评价的QoE评价体系.

\section{3 基于大数据挖掘的低能耗绿色通信}

通过对移动大数据的分析, 可以获取用户行为 习惯、业务特征、移动性等相关信息，并在此基础上 对用户行为趋势做出预测, 从而为无线网络低能耗 的绿色通信提供重要参考依据.

基于大数据的分析结果，网络侧可以更精准地 掌握不同地理区域的用户分布及业务需求，完成多样 化基站的智能部署，从而有效实现匹配用户业务需求 的动态资源分配; 结合用户的移动性等信息(如位置 信息、移动速度、移动方向等)实现超密集异构网络中 不同层级基站的动态激活与休眠、资源共享、协作传 输等，从而降低网络整体能耗，提高传输效率.

与此同时，终端侧可以基于自身业务特性和行 为习惯, 选择合适的接人网络, 辅助优化网络间协 作; 针对具有相似特征的业务需求或地理位置临近 的终端组, 还可以通过终端间直接通信(D2D)的方式 完成信息交互与推送，从而节省网络资源，降低发射 功率, 实现低能耗通信.

\section{4 结论}

移动大数据时代，无线网络在资源管控、数据安 全、网络能耗方面面临着新的挑战. 但移动大数据是 友好可控的, 是无线通信发展的助推剂, 给无线通信 带来了新的发展机遇. 无线网络可以充分借鉴互联 网数据挖掘的理论与方法, 实现网络的灵活部署、无 线资源的优化配置和低能耗绿色通信. 同时, 移动运 营商可充分利用自身的数据优势, 充分挖掘大数据 的隐性价值, 不断提升业务营销水平和服务质量, 实 现跨领域融合，更多的服务于科技进步和国家政策 等方面.

\section{参考文献}

1 Li G J. Scientific value of big data research (in Chinese). China Comput Soc Newslett, 2012, 8: 8-15 [李国杰. 大数据研究的科学价值. 中国计算机学会通讯, 2012, 8: 8-15]

2 Li G J, Cheng X Q. Big data research: Key strategic areas for future technological and economic social development-Status and scientific consideration of big data (in Chinese). Bull Acad Sci, 2012, 6: 647-657 [李国杰, 程学旗. 大数据研究: 未来科技及经济社会发展 的重大战略领域一大数据的研究现状与科学思考. 中国科学院院刊, 2012, 6: 647-657]

3 The 34th Statistical Report of the Development of Internet in China (in Chinese). China Internet Network Information Center, 2014 [第 34 次中国互联网络发展状况统计报告. 中国互联网络信息中心, 2014]

4 2013-2018 Global Mobile Data Traffic Forecast Update (in Chinese). Cisco Visual Networking Index (VNI), 2013 [2013 2018 年全球移 动数据流量预测更新. 思科视觉网络指数, 2013]

5 Li W, Lang B. A tetrahedral data model for unstructured data management. Sci China Inf Sci, 2010, 53: 1497-1510 
6 Andrews J G, Buzzi S, Wan C, et al. What will 5G be? IEEE J Sel Area Comm, 2014, 32: 1065-1082

7 Scenarios and requirements for small cell enhancements for E-UTRA and E-UTRAN (Release 12), 3GPP, TR 36.932 v12.1.0, 2013

8 Small cell enhancements for E-UTRA and E-UTRAN-Physical layer aspects, 3GPP, TR 36.872, 2013

9 Study on Small Cell enhancements for E-UTRA and E-UTRAN-Higher layer aspects, 3GPP, TR 36.842, 2013

10 Feng W. The opportunities and challenges for information security in the era of big data (in Chinese). Chin Tech Inv, 2012, 34: 49-53 [冯 伟. 大数据时代面临的信息安全机遇和挑战. 中国科技投资, 2012, 34: 49-53]

11 Feng D G, Zhang M, Li H. Big data security and privacy protection (in Chinese). Chin J Comput, 2014, 37: 246-258 [冯登国, 张敏, 李 昊. 大数据安全与隐私保护. 计算机学报, 2014, 1: 246-258]

12 Wang L, Meng X F. An overview of privacy protection of position big data (in Chinese). J Softw, 2014, 4: 693-712 [王璐, 孟小峰. 位置 大数据隐私保护研究综述. 软件学报, 2014, 25: 693-712]

13 Wyner A. The wire-tap channel. Bell Sys Tech J, 1975, 54: 1355-1387

14 Oggier F, Hassibi B. The secrecy capacity of the MIMO wiretap channel. IEEE Trans Inform Theory, 2011, 57: 4961-4972

15 Karpovsky M, Wang Z. Design of strongly secure communication and computation channels by nonlinear error detecting codes. IEEE Trans Comput, 2014, 63: 2716-2728

16 Zhang J, Gursoy M C. Collaborative relay beamforming for secrecy. In: Proceeding of 2010 IEEE International Conference on Communications (ICC2010). Cape Town: IEEE, 2010. 1-5

17 Chung P J. Green radio-the case for more efficient cellular base stations (slides). UK-Taiwan ICT Workshop: Smart \& Green Communications, 2009

18 Takaishi D, Nishiyama H, Kato N, et al. Towards energy efficient big data gathering in densely distributed sensor networks. IEEE Trans Emerg Topics Comput, 2014, 2: 388-397

19 Zhang Q W. How to deal with the challenges of traffic in data backbone network (in Chinese). Chin Manage Inform, 2012, 15: 73-74 [张 庆武. 如何应对数据骨干网流量的挑战. 中国管理信息化, 2012,6: 73-74]

20 China Mobile Research Institute. C-RAN Radio Access Network Evolution to Green: White Paper (in Chinese). 2010 [中国移动通信研究 院. C-RAN 无线接人网绿色演进: 白皮书. 2010]

21 Shi F, Wu H J. Trends of new intelligent pipeline technology based on SDN and NFV (in Chinese). Telecomm Net Tech, 2013, 3: 1-4 [史 凡, 吴宏建. 基于 SDN 和 NFV 的新型智能管道技术发展趋势. 电信网技术, 2013, 3: 1-4]

22 Tan Y, Wang H, Zhou Q H. Research of clustering algorithm in data mining (in Chinese). Net Sec Tech App, 2014, 1: 65-66 [覃艳, 王洪, 周全华. 数据挖掘中聚类算法的研究. 网络安全技术与应用, 2014, 1: 65-66]

23 Lin C, Hu J, Kong X Z. An overview of QoE-based modeling and evaluation methods (in Chinese). Chin J Comput, 2012, 35: 1-15 [林闯, 胡杰, 孔祥震. 用户体验质量 $(\mathrm{QoE})$ 的模型与评价方法综述. 计算机学报, 2012, 35: 1-15]

\title{
Opportunities and challenges of wireless networks in the era of mobile big data
}

\author{
ZHANG Ping, CUI QiMei, HOU YanZhao \& XU Jin \\ Beijing University of Posts and Telecommunications, Beijing 100876, China
}

With the rapid development of information and communication technologies such as mobile Internet, cloud computing, and the Internet of Things, the information society has stepped into the networked big data era. Smart terminals, widely applied in everyday life, are driving the explosive demands of wireless data services. In the era of mobile big data, wireless networks face with many challenges owing to the specific characteristics of big data such as high volume, variety, and non-uniform distribution in the time and space domains. And new approaches are required to address these new challenges. On the one hand, new spectrum technology, transmission technology, and intelligent networking offer promising evolution directions. On the other hand, mining the information contained in the low-density mobile big data, could be a new way to optimize current wireless networks. Data mining theory incorporated with mobile Internet can be applied in a wireless network to realize flexible networking, optimization of radio resource configuration, and green communication.

mobile big data, wireless networks, opportunities and challenges, network deployment, resource allocation forecast doi: 10.1360/N972014-01165 